\title{
Churg-Strauss syndrome leading to small bowel infarction: An unusual case of abdominal pain in a young patient
}

Sunil Sookram MD, Camille Hancock-Friesen MD, JP Ferguson MD FRCPC, Terry Sosnowski MD FRCPC, Laurie JM Russell MD FRCPC

S Sookram, C Hancock-Friesen, JP Ferguson, T Sosnowski, LJM Russell. Churg-Strauss syndrome leading to small bowel infarction: An unusual case of abdominal pain in a young patient. Can J Gastroenterol 1998;12(3):219-222. A 33-year-old man with a history of severe asthma presented to the emergency department with a week-long history of severe unrelenting abdominal pain, nausea and decreased appetite. He was admitted to hospital, and routine gastrointestinal investigations were performed, which did not elucidate the cause of his abdominal pain. Exploratory laparotomy demonstrated patchy infarction of the entire small bowel, characteristic of Churg-Strauss syndrome. The patient subsequently underwent 12 separate laparotomies to salvage surviving small bowel. The patient is maintained on total parenteral nutrition.

Key Words: Asthma, Churg-Strauss syndrome, Mesenteric ischemia, Vasculitis

\section{Infarctus du grêle consécutif à un syndrome} de Churg-Strauss : cas inhabituel de douleurs abdominales che $z$ un jeune patient

RÉSUMÉ : Un homme de 33 ans, présentant des antécédents d'asthme grave, se présente au service des urgences pour une douleur abdominale, des nausées et une réduction de l'appétit persistantes et graves depuis une semaine. Il est hospitalisé et les épreuves gastrointestinales de routine sont effectuées, mais ne permettent pas d'identifier la cause de sa douleur abdominale. Une laparotomie exploratoire a permis d'identifier un infarctus en plaques de tout l'intestin grêle, caractéristique d'un syndrome de Churg-Strauss. Le patient a par la suite subi 12 laparotomies distinctes pour sauver les portions du grêle restées fonctionnelles. Le patient reçoit à l'heure actuelle une nutrition parentérale totale.
$\mathrm{P}$ atients frequently present to the emergency department with abdominal pain. Clinicians must assess patients and determine the diagnosis and treatment, while primarily excluding the possibility of rare life-threatening conditions. Intestinal ischemic disease, which is uncommon but potentially serious, is a surgical emergency that is diagnostically elusive. Although often presenting in the elderly with multiple risk factors (ie, atrial fibrillation or congestive heart failure), it is quite a rare phenomenon in young, otherwise healthy, patients. Vasculitic disease of the mesenteric vessels is a cause of only $2 \%$ of mesenteric ischemia (1). Various rheumatological diseases have systemic manifestations that 
can affect the vessels of the gut, including polyarteritis nodosa, Wagner's granulomatosis, systemic lupus erythematosis, rheumatoid arthritis and Churg-Strauss syndrome. We present a case of a 33-year-old male who was afflicted with the rare intestinal manifestations of Churg-Strauss syndrome.

\section{CASE PRESENTATION}

A 33-year-old male, with a five-year history of severe asthma requiring daily oral prednisone use, presented to the emergency department in a large urban referral centre. He complained of a six-day history of severe intractable periumbilical abdominal pain. The pain began about 2 to $3 \mathrm{~h}$ following ingestion of some tuna, which was subsequently discarded by his wife because it was malodourous. Other family members consumed the tuna but did not suffer any similar symptoms. The pain was described as sharp, nonradiating and constant. It was severe enough to prevent him from sleeping on several occasions. He experienced nausea but no vomiting. Despite anorexia, he was able to tolerate fluids and occasional food products. He had experienced a few episodes of diarrhea since the onset of pain; otherwise, his stool was normal. He denied any melena or blood per rectum. He admitted to occasional fever and chills. Since the onset of symptoms he had been assessed twice in ambulatory care settings and given pain medication, which was not helping. He was sent for an upper gastrointestinal series, which was reported as normal. In addition, he had been assessed two days earlier in an emergency department and diagnosed with gastroenteritis. At that time, he was given fluids intravenously, dimenhydrinate and meperidine, and improved; he was subsequently discharged home.

Our patient was previously well, except for asthma, and was free of abdominal complaints. He denied having any contacts with anyone who was experiencing similar problems. There was no recent travel before his illness. He reported no previous abdominal surgery, and he denied any recent antibiotic use. He denied recent loss of weight, or tenesmus, rash or joint pain. There was no family history of inflammatory bowel disease. This young man had suffered from severe asthma over the past five years that was complicated by aspergillosis, which was discovered during bronchoscopy three years previously. As a result of intractable asthma, he had been on long term oral prednisone. At the time of presentation, he took $10 \mathrm{mg}$ twice daily; however, there were periods where he needed doses of up to $100 \mathrm{mg} /$ day. As a result of long term prednisone use, he developed insulin-dependent diabetes mellitus. Regular monitoring revealed blood glucose levels consistently in the range of 7 to $10 \mathrm{mmol} / \mathrm{L}$.

On examination, this patient was in moderate distress. He was an obese man with many cushingoid features. His vital signs were as follows: heart rate 120 beats/min, temperature $37^{\circ} \mathrm{C}$ and blood pressure $140 / 90 \mathrm{mmHg}$. Respiratory examination showed wheezing bilaterally. Bowel sounds were present, and there was no rebound, guarding or percussion tenderness. He experienced some pain on deep palpation periumbilically. There was no evidence of any hernias, and the genitourinary examination was normal. Rectal ex- amination revealed no masses. There was stool in the rectum, which tested negative for occult blood. The remainder of the physical examination was unremarkable.

Initial laboratory tests done in the emergency department revealed a normal hemoglobin and platelet count. The white blood cell count was $17.4 \times 10^{9} / \mathrm{L}$. Cell differentials were as follows: neutrophils $73 \%$, lymphocytes $3 \%$ and eosinophils $22 \%$. Electrolyte levels were as follows: sodium $139 \mathrm{mmol} / \mathrm{L}$, potassium $3.9 \mathrm{mmol} / \mathrm{L}$ and chloride $100 \mathrm{in} \mathrm{mmol} / \mathrm{L}$. Creatinine level was $81 \mu \mathrm{mol} / \mathrm{L}$, glucose $7 \mathrm{mmol} / \mathrm{L}$, and anion gap 21. Liver enzyme levels were as follows: alanine aminotransferase $55 \mathrm{U} / \mathrm{L}$, aspartate aminotransferase $26 \mathrm{U} / \mathrm{L}$, alkaline phosphatase $242 \mathrm{U} / \mathrm{L}$ and lipase $30 \mathrm{U} / \mathrm{L}$. Liver function tests showed a protein level of $75 \mathrm{~g} / \mathrm{L}$ and albumin $35 \mathrm{~g} / \mathrm{L}$. Urinalysis was unremarkable.

Initial radiological studies included a chest and abdominal series. Diffuse interstitial markings were demonstrated on chest radiography. Radiography of the abdomen revealed barium from the previous upper gastrointestinal series, and the gas pattern was interpreted as normal. There was no evidence of intra-abdominal disease.

Because of the severity of his pain the patient was admitted for investigation and treatment. He received narcotic analgesia for the pain. An abdominal ultrasound was normal with no evidence of cholestatic disease or pancreatitis. Stool was sent for culture and sensitivity, ova and parasites, and Clostridium difficile toxin. The laboratory reported that all results were normal.

Following admission, the patient was ambulatory yet was constantly requesting additional pain medication. A gastroscopy performed on his fourth day in hospital revealed diffuse superficial erosive gastritis that was felt to be not significant enough to account for his pain. There was no evidence of peptic ulcer disease or inflammatory bowel disease changes up to the ligament of Trietz. Later that day, his pain increased, but there was no change on abdominal examination. On the fifth day of admission, a repeat abdominal series revealed several mildly dilated air and fluid-filled loops of small bowel. The radiologist reported some mild mucosal thickening within small bowel loops. Repeated blood work showed that his white blood cell count had increased to $29.5 \times 10^{9} / \mathrm{L}$. A surgical consultation was obtained, and a decision was made to proceed with an exploratory laparotomy.

At laparotomy, an infarcted omentum was discovered, and there were multiple patchy regions of ischemia throughout the small bowel extending from the jejunum through the entire ileum. Some regions showed transmural bowel damage.

An abdominal aortogram (Figure 1) was performed after the laparotomy. The aortogram showed segmental stenosis of the distal superior mesenteric artery, and ileocolic and jejunal arteries, which was thought to be consistent with a vasculitis. Biopsy specimens obtained intraoperatively demonstrated multiple regions of transmural infarction and ischemia. Many of the small blood vessels contained eosinophilia, and multiple sections of the infarcted omentum 
contained acute necrotizing granulomatous vasculitis. Pathological features were in keeping with Churg-Strauss syndrome.

The patient went to the intensive care unit postoperatively and subsequently underwent 12 laparotomies and bowel resections in an attempt to maximize the preservation of viable small bowel. He was discharged home four months later with supplemental parenteral nutrition, with hopes that his jejunostomy would later be reconnected to distal surviving bowel.

\section{DISCUSSION}

Rheumatological disorders causing vasculitis are potentially serious and often difficult to diagnose. Many end organs are susceptible to damage and rarely effectively managed before the ischemic insult occurs. There are reports of cerebrovascular and cardiac insults secondary to vasculitic injury to smaller blood vessels. Mesenteric vasculitides are rare causes of ischemia. Churg-Strauss syndrome is a member of the family of systemic vasculitides, whose diagnosis is difficult to elucidate in the acute setting.

Churg-Strauss is also known as allergic granulomatosis or angiitis; it is quite rare and has no known cause. The condition is often found in patients having a history of asthma or allergy. It characteristically affects middle-aged people. Men are more commonly afflicted than women. Common findings are fever, malaise and weight loss. The disease often manifests in three phases. First, a prodromal phase exists with persistent allergic disease, ie, rhinitis or asthma. The next stage is characterized by the appearance of peripheral blood and tissue eosinophilia. Finally, there may be remittance or recurring eosinophilic infiltrative disease over several years before the onset of life-threatening systemic vasculitis (2). Churg-Strauss sufferers often complain of asthma that is refractory to treatment and worsening despite aggressive management by their physicians. There are often cutaneous manifestations including petechiae, purpura or ulceration. Peripheral neuropathy, glomerulonephritis and mesenteric ischemia can occur (3).

Several series have shown gut ischemia occurring in vasculitic disease (1,4-6). In a large series of Churg-Strauss patients, one of 165 patients was found to have intestinal infarction (6). However, other abdominal signs and symptoms were present $(31 \%)$, including peritonitis $(5 \%)$, duodenal ulcer $(2 \%)$ and gastrointestinal hemorrhage $(7 \%)$, some of which may be due to ischemic insult (6).

Churg-Strauss is rarely diagnosed before laparotomy or autopsy (4). Pathology includes eosinophilic granulomatosis involving the blood vessels. Angiitis and extramural necrotizing microgranulomas with eosinophilic infiltrates are found on biopsy, which is consistent with findings from our patient's specimens, which were obtained intraoperatively $(3,7)$. In the lungs, patchy or nodular infiltrations are often seen radiologically (3). However, these findings were not seen with the present case. Hematological examination should show eosinophilia of greater than 10\% (3). Serum complement is usually normal (3). Other rheumatological

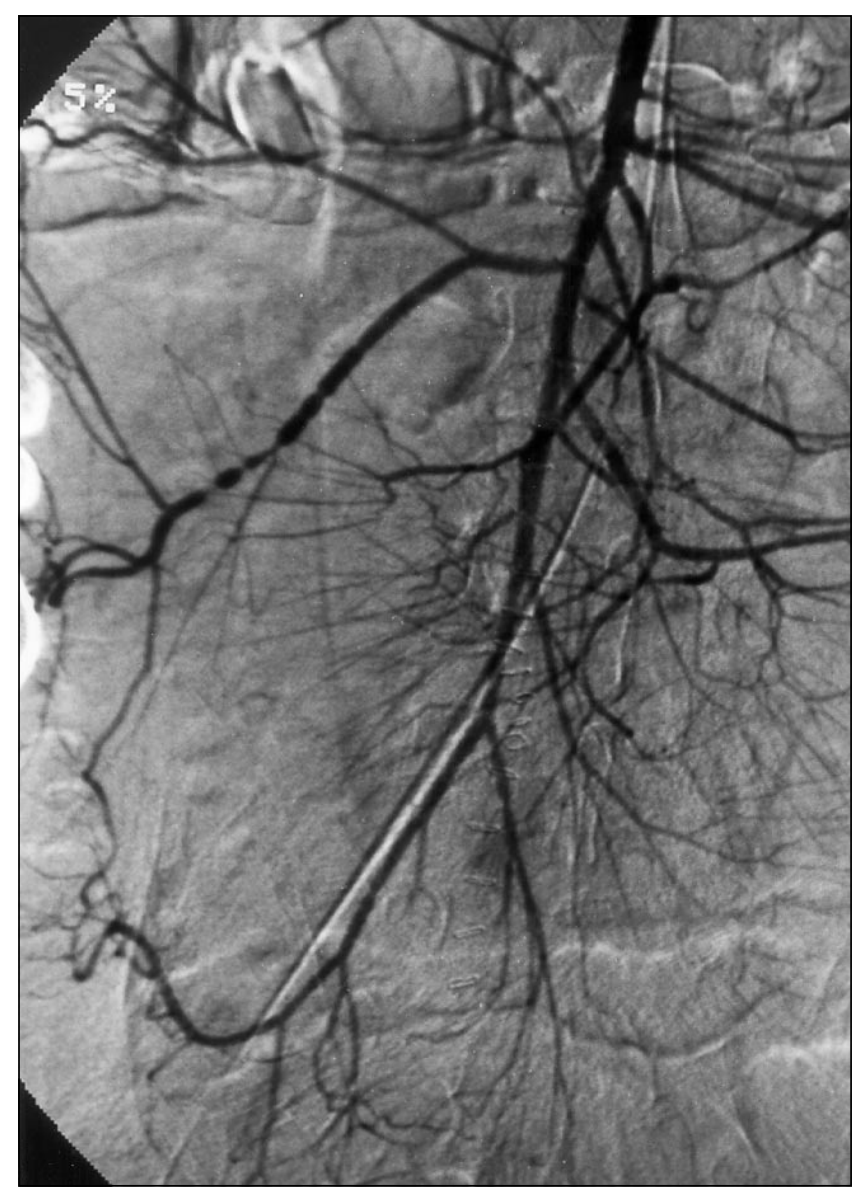

Figure 1) Arteriogram showing multiple stenotic sites within the ileocolic artery and other mesenteric vessels

markers are often elevated. This disease should show elevations of erythrocyte sedimentation rate (ESR), rheumatoid factor and immunoglobulin (Ig) levels $(1,3,4)$. Our patient exhibited an elevation of IgM antibodies and an elevated ESR of $55 \mathrm{~mm} / \mathrm{h}$, and antineutrophil cytoplasmic antibodies assay was negative and rheumatoid factor was elevated at $418 \mathrm{Ku} / \mathrm{h}$.

As mentioned earlier, Churg-Strauss is often found in patients having a history of severe asthma or rhinitis. On bronchoscopy performed three years previously, pathological aspergillosis was diagnosed. Thus, the patient's asthma was never attributed to a rheumatological cause. Nor were any systemic signs of vasculitis present in the patient's history or during physical examination. The patient required chronic oral corticosteroid use to control his asthma. The initial elevation of the white blood cell count could have been attributed to steroid use, while the eosinophilia was initially attributed to an allergic asthma process. Even though steroid use should decrease the amount of inflammatory reaction, its use in smaller doses does not preclude vasculitic disease. Substantial eosinophilia greater than $10 \%$ needs to be investigated and not attributed to steroid use or allergic asthma.

This patient was managed with multiple laparotomies in an attempt to conserve the maximum length of viable small 


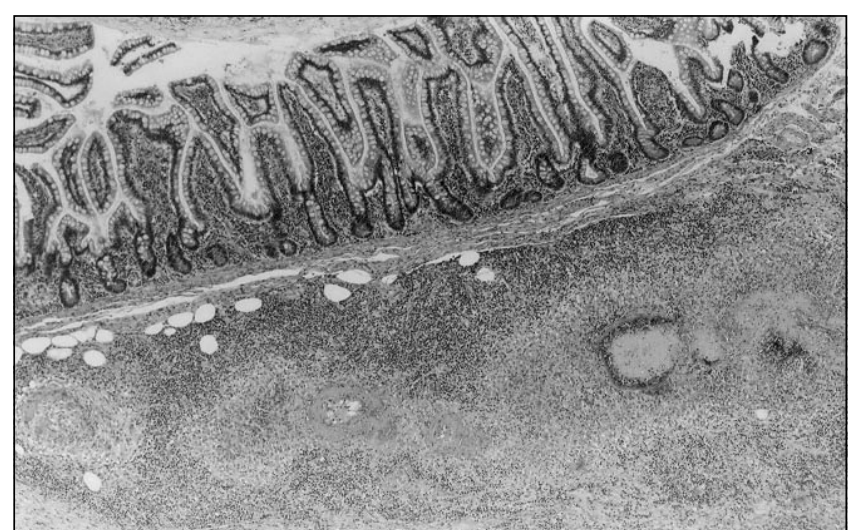

Figure 2) Micrograph showing submucosa of small bowel and intense acute inflammation around damaged submucosal vessels. There is dense eosinophilic cuffing around vessels

bowel. He was heparinized postoperatively and treated with pulsed cyclophosphamide and intravenous methylprednisolone. Because the patient required several operations while receiving concurrent therapy, pathological specimens obtained intraoperatively show the various stages of healing of Churg-Strauss vasculitis (Figures 2,3).

Appropriate management of Churg-Strauss may include the use of high dose steroids in combination with cytotoxic medication such as cyclophosphamide. Bacon (5) reported decreases in both mortality and relapse rate in more than 100 cases of systemic necrotizing vasculitis with intermittent pulsed cyclophosphamide therapy, methylprednisolone and plasma exchange (8).

Churg-Strauss syndrome is one of several vasculitic diseases that can lead to mesenteric ischemia. The presence of pain out of proportion with physical findings in any patient with no risk factors for ischemia should alert the clinician to the possibility of mesenteric vasculitis among the differential diagnoses.

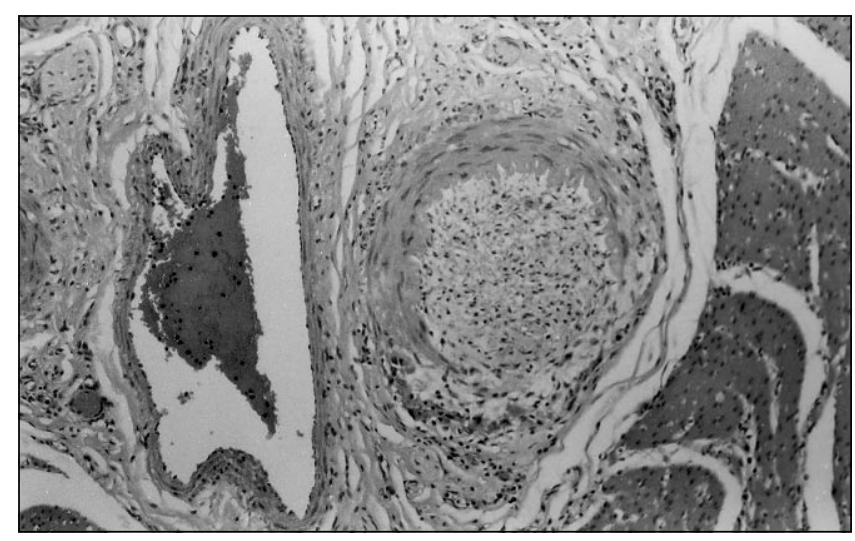

Figure 3) Micrograph taken during healing phase. There is an artery with organized thrombus and recanalization present. The surrounding vein is normal

ACKNOWLEDGEMENTS: The authors thank Dr Brian H Rowe for his assistance in preparing this manuscript.

\section{REFERENCES}

1. Schoretsanitis GN, Wakely D, Maddox T, Wastell C. A case of Churg-Strauss vasculitis complicated by small bowel necrosis. Postgrad Med J 1993;69:828-31.

2. Specks U, DeRemee R. Gramulomatous vasculitis, Wagner's granulomatosis and Churg-Strauss syndrome. Rheum Dis Clin North Am 1990;16:377-97.

3. Schumacher R, ed. Primer on the Rheumatic Diseases, 10th edn. Atlanta: Arthritis Foundation, 1993:145-9.

4. Lanham JG, Elkon KB, Pusey CD, Hughes GR. Systemic vasculitis with asthma and eosinophilia: a clinical approach to the Churg-Strauss syndrome. Medicine (Baltimore) 1984;63:65-81.

5. Bacon PA. Vasculitis - clinical aspects and therapy. Acta Med Scand Suppl 1987;715:153-63.

6. Guillevin L, Huong DLT, Godern P, Jais P, Wechsler B. Clinical findings and prognosis of polyarteritis nodosa and Churg-Strauss angiitis: a study of 165 patients. Br J Rheumatol 1988;27:258-64.

7. Chumbley LC, Harrison EG, DeRemee RA. Allergic granulomatosis and angiitis (Churg-Strauss syndrome). Mayo Clin Proc 1977;52:477-84.

8. Conn DL. Role of cyclophosphamide in treatment of polyarteritis nodosa. J Rheumatol 1991;18:567-74. (Edit) 


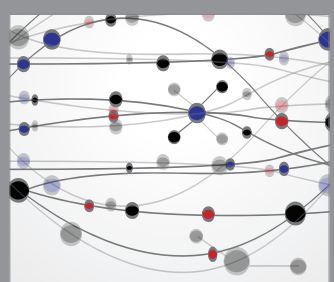

The Scientific World Journal
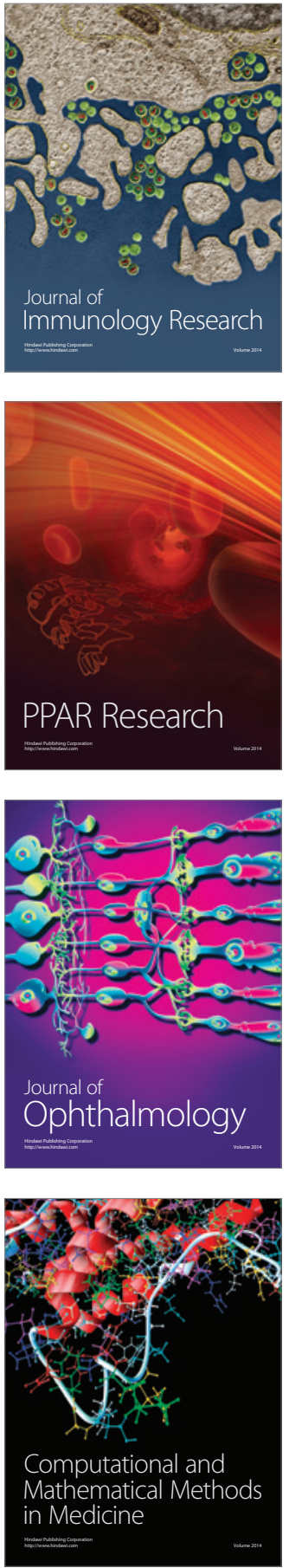

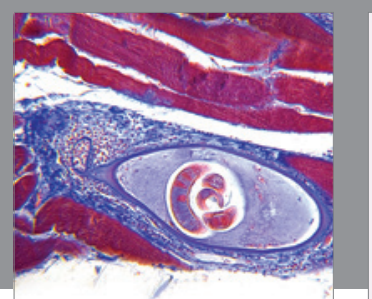

Gastroenterology Research and Practice

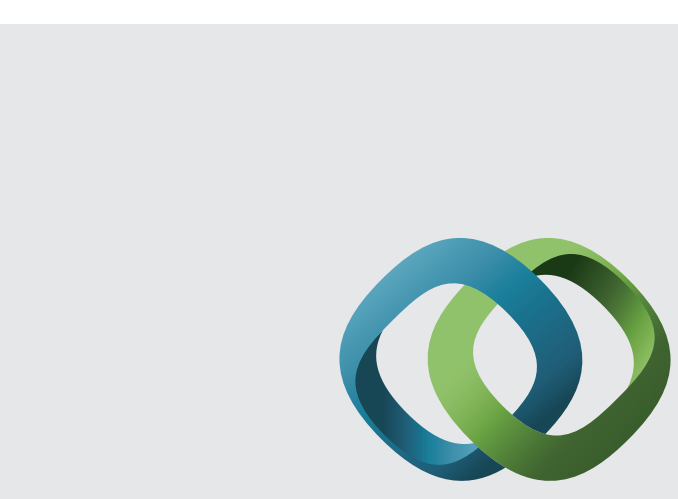

\section{Hindawi}

Submit your manuscripts at

http://www.hindawi.com
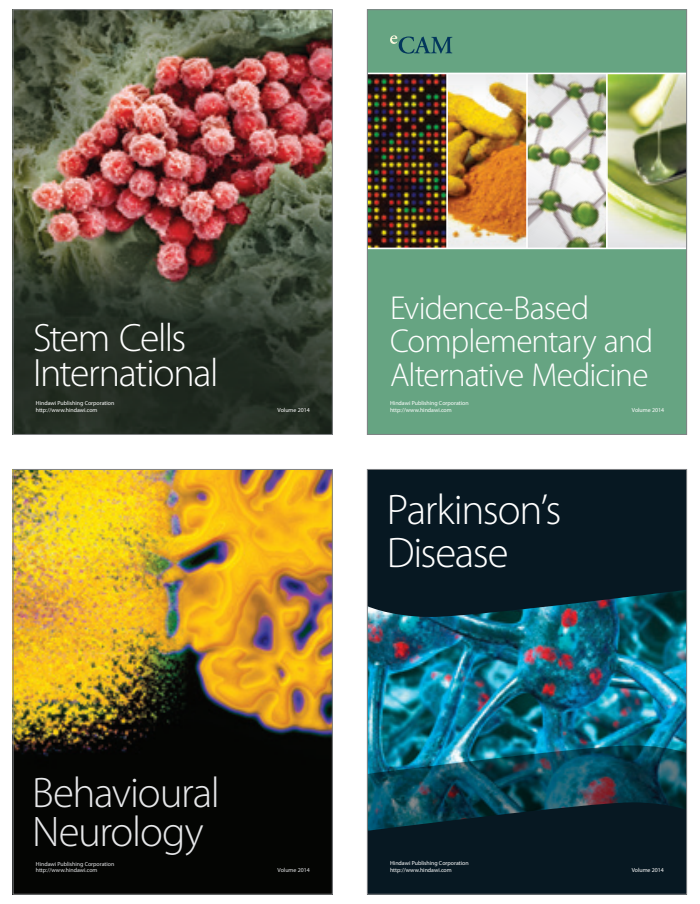
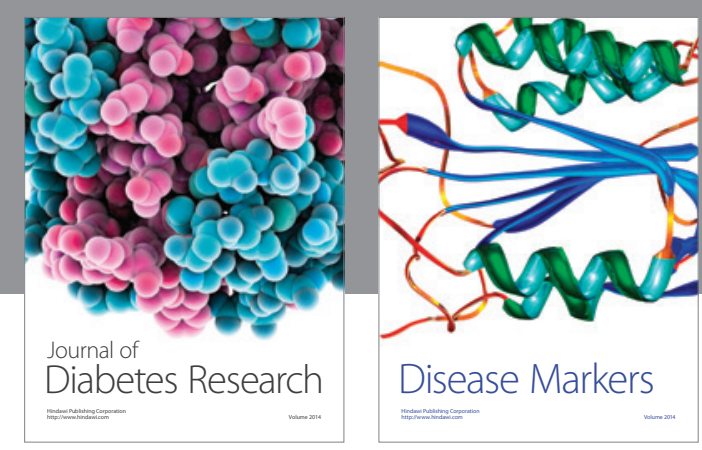

Disease Markers
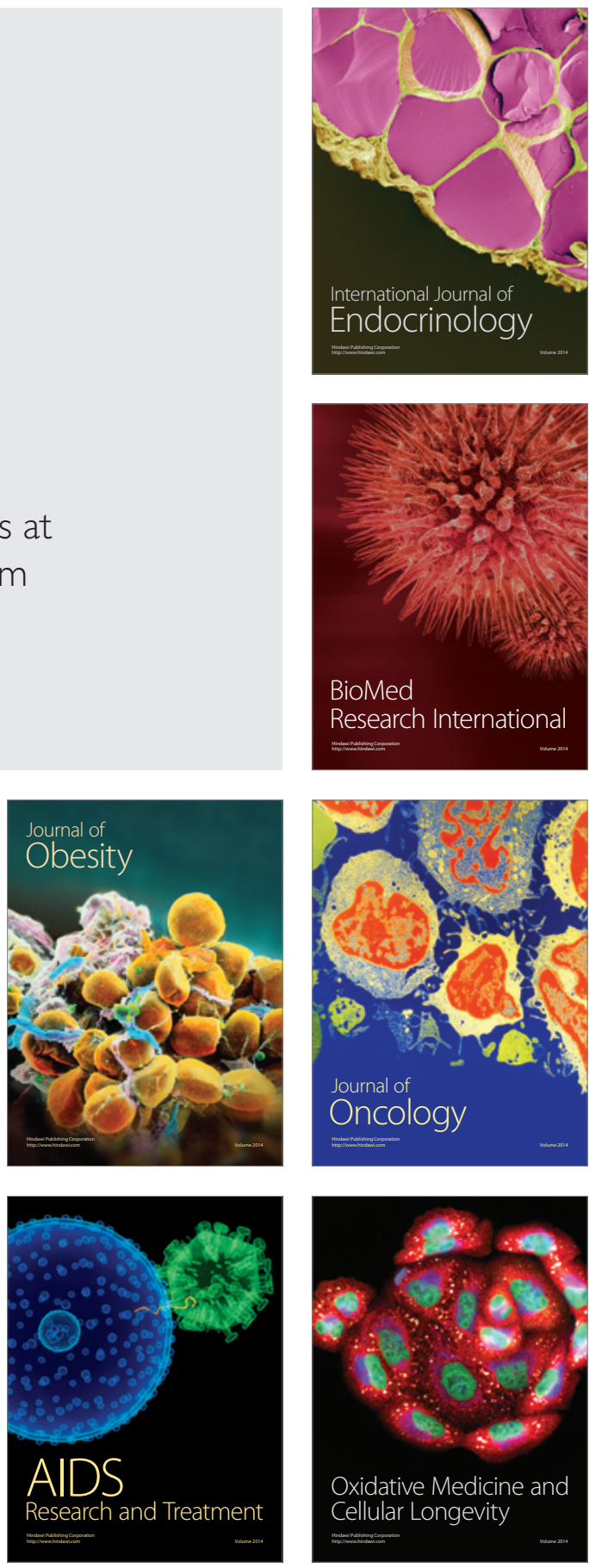